\title{
SIMULAÇÃO NUMÉRICA DE ESCOAMENTO PARTICULADO APLICADO AO PREENCHIMENTO DE CANAL FRATURADO
}

\author{
M.V. BARBOSA ${ }^{1 *}$, F. C. DE LAI ${ }^{1}$, S. L. M. JUNQUEIRA ${ }^{1 \#}$ \\ ${ }^{1}$ Universidade Tecnológica Federal do Paraná (UTFPR), Centro de Estudos em Reologia e Fluidos \\ Não Newtonianos (CERNN), Programa de Pós-Graduação em Engenharia Mecânica e de Materiais \\ (PPGEM ) \\ e-mail: *marcoscp2@gmail.com ; " silvio@utfpr.edu.br
}

\begin{abstract}
RESUMO
No presente trabalho, uma análise numérica do preenchimento de um canal vertical com fratura transversal é apresentada. A abordagem escolhida para o preenchimento consiste em adicionar material particulado ao escoamento para promover a deposição de partícula no interior da fratura, reduzindo a fuga de fluido nessa região. A análise é realizada através de uma abordagem euleriana para o fluido e lagrangiana para as partículas. Para efetuar o acoplamento entre as fases e realizar o cálculo das colisões entre as partículas os modelos DDPM (Dense Discrete Phase Model) e DEM (Discrete Element Method) são combinados. O resultado do processo de preenchimento, estudado através da variação da quantidade de partículas injetadas no canal, é analisado através das características geométricas do leito de partículas formado no interior da fratura, da vazão de fluido sendo perdido pela fratura e através do monitoramento da pressão na entrada do canal devido ao processo de injeção. Os resultados mostram que a influência da quantidade de partículas injetadas exerce maior influência sobre o tempo necessário para realizar o preenchimento. Assim, a concentração reduz o tempo de preenchimento, porém implica no aumento da pressão na região de entrada do canal.
\end{abstract}

\section{INTRODUÇÃO}

O escoamento bifásico do tipo líquidosólido é encontrado em diversas aplicações como separadores ciclônicos, leitos fluidizados, transporte de sedimentos, entre outros (LOTH, 2010)

Estudar o escoamento particulado de forma experimental é uma tarefa muitas vezes complexa, principalmente do ponto de vista de desenvolvimento de sensores para a mensuração de propriedades relativas à fase sólida. Sendo assim, a simulação numérica de tal categoria de escoamento é utilizada frequentemente como alternativa. (YEOH E TU, 2009)
Em simulações computacionais do escoamento particulado, duas abordagens podem ser realizadas, com relação à fase sólida: a abordagem euleriana ou lagrangiana.

A abordagem euleriana considera a fase sólida como um meio contínuo com a habilidade de interpenetrar e interagir com o fluido ao seu redor. O rastreio das partículas é feito através da fração volumétrica da fase sólida (GIDASPOW, 1994).

$\mathrm{Na}$ abordagem lagrangiana as partículas são tratadas de forma discreta e rastreadas individualmente ao longo de todo domínio numérico computacional (PATANKAR E JOSEPH, 2001)

Cada modelo possui seus pontos fortes e fracos: no modelo euleriano o tratamento de 
um número elevado de partículas é possível, porém existe a necessidade de determinar relações de fechamento para garantir um balanço eficaz nas equações de conservação; no modelo lagrangiano as equações de fechamento não são necessárias, mas simular uma quantidade elevada de partículas pode se tornar um processo custoso, do ponto de vista computacional (GIDASPOW, 1994).

A escolha de cada modelo depende de um grande número de variáveis, sendo as principais aquelas que demandam informações específicas sobre o deslocamento de cada partícula durante o estudo do fenômeno escolhido.

Neste trabalho, o escoamento líquidosólido é utilizado para preencher um canal fraturado. Tal problema possui diversas aplicações, em especial na indústria do petróleo, seja no fraturamento artificial de reservatórios ou na redução/eliminação do problema de perda de circulação (DE LAI, 2013; BARBOSA, 2015).

A descrição matemática da fase discreta é feita através de uma modelagem do tipo lagrangiana, já que se deseja observar o transporte de partículas do canal em direção à fratura.

O processo de preenchimento da fratura é analisado através da concentração de partículas, determinada a partir da quantidade de partículas injetadas por segundo ao fluido, $\mathrm{N}_{p, \text { inj }}$.

As variáveis de interesse do problema consistem na vazão de fuga devido à presença da fratura $Q_{\text {fuga }}$, na pressão de entrada no canal fraturado $P_{e n t}$ e nas características geométricas do leito de partículas formado no interior da fratura como: posição inicial $h_{p c t, i}$, comprimento do leito $h_{p c t}$ e preenchimento vertical da fratura $e_{\%, F R}$.

Além disso, o tempo necessário para que a fratura seja preenchida $t_{\text {est }}$ também é monitorado.

\section{CARACTERIZAÇÃO DO PROBLEMA}

O problema a ser estudado consiste no escoamento vertical em um canal com a presença de uma fratura, assim como mostrado na Figura 1.

Figura 1 - Geometria do canal fraturado

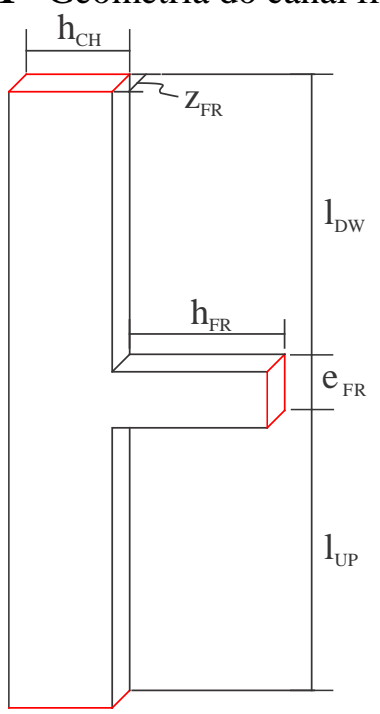

Na Figura 1 a fratura no domínio computacional é definida através de seu comprimento $\left(h_{F R}\right)$ e de sua espessura $\left(e_{F R}\right)$. O comprimento do canal à montante e à jusante da fratura é dado por $l_{U P}$ e $l_{D W}$, respectivamente. A abertura do canal é definida por $h_{C H}$ e o sistema como um todo possui componente na direção $\mathrm{z}, \mathrm{z}_{F R}$.

O canal e a fratura são considerados impermeáveis. A parede final da fratura $\left(x=h_{F R}\right)$ é considerada permeável apenas à passagem de fluido. Dessa forma, existe a perda de fluido associada à presença da fratura no canal.

Para selar a fratura, é empregada a injeção de material particulado no canal. As partículas serão transportadas durante toda a extensão do canal, até a entrada da fratura, onde as mesmas são depositadas por ação da gravidade.

A dificuldade na caracterização de tal problema reside na necessidade de determinar as corretas condições de contorno a serem aplicadas durante a fase de injeção de 
partículas. Em vista disso, De Lai (2013) propôs utilizar a quantidade de fluido sendo perdido através da fratura como base para a determinação das pressões associadas em cada superfície de saída de fluido, i.e., saída do canal fraturado e saída da fratura.

Para tanto, o autor se valeu de uma condição de velocidade prescrita na entrada do canal fraturado, $U_{\beta, C H, i}$, determinado a partir do número de Reynolds desejado no canal, mostrado na Eq. (1), sendo $\rho_{\beta}$ a massa específicia e $\varepsilon_{\beta}$ a viscosidade do fluido.

$$
U_{\beta, C H, i}=\frac{\operatorname{Re} \mu_{\beta}}{\rho_{\beta} h_{C H}}
$$

A mesma metodologia desenvolvida por De Lai (2013) é aplicada neste trabalho.

\section{MODELO NUMÉRICO}

O modelo Dense Discrete Phase Model, proposto por Popoff e Braun (2007), é utilizado neste trabalho. A descrição da fase contínua (fluido) é feita através de uma abordagem euleriana, enquanto as partículas (fase discreta) são descritas através de uma abordagem lagrangiana.

A formulação matemática consiste em dois conjuntos de equações: um para o fluido e outro para as partículas. Nesse estudo, o fluido é considerado newtoniano em um escoamento incompressível e isotérmico. As equações para a fase contínua são dadas pela conservação da massa, Eq. (2) e pelo balanço de quantidade de movimento, Eq. (3).

$$
\begin{aligned}
& \frac{\partial \varepsilon_{\beta} \rho_{\beta}}{\partial t}+\nabla \cdot\left(\varepsilon_{\beta} \rho_{\beta} \boldsymbol{u}_{\beta}\right)=0 \\
& \frac{\partial\left(\varepsilon_{\beta} \rho_{\beta} \boldsymbol{u}_{\beta}\right)}{\partial t}+\nabla \cdot\left(\varepsilon_{\beta} \rho_{\beta} \boldsymbol{u}_{\beta} \boldsymbol{u}_{\beta}\right)=-\varepsilon_{\beta} \nabla \boldsymbol{p}_{\beta} \\
& \quad+\nabla \cdot\left(\varepsilon_{\beta} \mu_{\beta} \nabla \cdot \boldsymbol{u}_{\beta}\right)+\varepsilon_{\beta} \rho_{\beta} \mathbf{g} \\
& \quad+\mathbf{F}_{D P M}+\mathbf{S}_{D P M}
\end{aligned}
$$

em que $t$ é o tempo, $\varepsilon_{\beta}$ é a fração volumétrica da fase contínua, $\boldsymbol{u}_{\beta}$ é o vetor velocidade, $\boldsymbol{p}_{\beta}$ é o gradiente de pressão, $\mathbf{g}$ é o vetor aceleração da gravidade. $\mathbf{F}_{D P M}$ representa a força de acoplamento entre as fases e $\mathbf{S}_{D P M}$ é o termo fonte devido ao descolamento do fluido em relação a entrada das partículas em um volume de controle.

Quanto à fase discreta, a posição de cada partícula é descrita em função da velocidade, como mostrado na Eq. (4). A segunda lei de Newton para o movimento, Eq (5), é aplicada para a determinação da velocidade da partícula.

$\frac{d \boldsymbol{x}_{p}}{d t}=\boldsymbol{u}_{p}$

$m_{p} \frac{d \mathbf{u}_{p}}{d t}=\mathbf{F}_{d}+\mathbf{F}_{g b}+\mathbf{F}_{p g}+\mathbf{F}_{v m}+\mathbf{F}_{l s}+\mathbf{F}_{D E M}$

Nas equações (4) e (5) a posição e velocidade da partícula é dada por $\boldsymbol{x}_{p}$ e $\boldsymbol{u}_{p}$, respectivamente. A massa da partícula é representada por $m_{p}, \mathbf{F}_{d}$ é a força de arrasto, $\mathbf{F}_{g b}$ representa $\mathbf{0}$ balanço entre força gravitacional e empuxo, $\mathbf{F}_{p g}$ representa a força devido ao gradiente de pressão, $\mathbf{F}_{v m}$ é a força devido à massa virtual, $\mathbf{F}_{l s}$ é a força de sustentação de Saffman e $\mathbf{F}_{D E M}=\mathbf{F}_{n}+\mathbf{F}_{t}$ é a força de colisão.

Na Tabela 1 vê-se a representação de cada força em que $\operatorname{Re}_{p}$ é o número de Reynolds das partículas, dado por $\operatorname{Re}_{p}=\rho_{\beta}\left|\boldsymbol{u}_{\beta}-\boldsymbol{u}_{p}\right| d_{p} / \mu_{\beta}$ e $\rho_{p}$ é a massa específica da partícula. $C_{l s}$ é a constante de Saffman (Li e Ahmadi 1992) e $C_{v m}$ é o coeficiente de massa virtual (Kendoush et al. 2007).

O coeficiente de arrasto, $C_{D}$ é calculado através do modelo proposto por Morsi e Alexander (1972), desenvolvido para apenas uma partícula esférica. O modelo foi escolhido por ser o único disponível no software comercial utilizado para escoamentos líquido-sólido. 
Tabela 1 - Expressões para a determinação das forças agindo sobre as partículas

\begin{tabular}{|c|c|c|}
\hline & Força & Equação \\
\hline & $\begin{array}{l}\text { ravitacional } \\
\text { e empuxo }\end{array}$ & $\mathbf{F}_{g b}=m_{p} \frac{\rho_{p}-\rho_{\beta}}{\rho_{p}} \mathbf{g}$ \\
\hline & Arrasto & $\mathbf{F}_{d}=\frac{3}{4} \frac{m_{p} \mu_{\beta}}{\rho_{p} d_{p}^{2}} C_{D} \operatorname{Re}_{p}\left(\boldsymbol{u}_{\beta}-\boldsymbol{u}_{p}\right)$ \\
\hline & $\begin{array}{l}\text { Uustentação } \\
\text { le Saffman }\end{array}$ & $\mathbf{F}_{l s}=C_{l s} m_{p} \frac{\rho_{\beta}}{\rho_{p}}\left(\nabla \times \boldsymbol{u}_{\beta}\right) \times\left(\boldsymbol{u}_{\beta}-\boldsymbol{u}_{p}\right)$ \\
\hline & Iassa virtual & $\mathbf{F}_{v m}=C_{v m} m_{p} \frac{\rho_{\beta}}{\rho_{p}} \frac{D}{D t}\left(\boldsymbol{u}_{\beta}-\boldsymbol{u}_{p}\right)$ \\
\hline & $\begin{array}{l}\text { Gradiente } \\
\text { de pressão }\end{array}$ & $\mathbf{F}_{p g}=m_{p} \frac{\rho_{\beta}}{\rho_{p}}\left(\boldsymbol{u}_{\beta} \nabla \cdot \boldsymbol{u}_{\beta}\right)$ \\
\hline \multirow{2}{*}{ Colisão } & Normal & $\mathbf{F} \boldsymbol{\lambda}=\left[k 2 \delta+\gamma\left(\boldsymbol{u}_{12} \cdot{ }_{12}\right)\right]_{12}$ \\
\hline & Tangencial & $\mathbf{F}_{t} \frac{\xi}{5}-\mu_{a}\left|\mathbf{F}_{n}\right|_{12}$ \\
\hline
\end{tabular}

A força de colisão $\mathbf{F}_{D E M}$ é uma combinação da força normal $\left(\mathbf{F}_{n}\right)$ e tangencial $\left(\mathbf{F}_{t}\right)$ gerada pela colisão entre as partículas. Ambas as forças são calculadas através do método dos elementos discretos (Discrete Element Method - DEM), proposto por Cundall e Strack (1979).

A força normal é calculada através de um modelo do tipo mola-amortecedor (Lunding, 1998), em que $k, \delta, \quad \gamma, \boldsymbol{u}_{12} \mathrm{e} \lambda_{12}$ são, respectivamente, a constante de rigidez, a sobreposição das partículas no momento da colisão, o coeficiente de amortecimento, a velocidade relativa entre as partículas e a direção normal de colisão. O coeficiente de amortecimento é calculado através da Eq. (6), com base em um coeficiente de restituição $\eta$. Na Eq. (6), $m_{12}$ representa a massa reduzida e $t_{c o l}$ é o tempo de colisão.

$\gamma=-2 \frac{m_{12}}{t_{c o l}} \ln \eta$

A força de colisão tangencial é calculada através do modelo de Coulomb com base em um coeficiente de atrito $\mu_{a}$ e na direção tangencial de colisão $\zeta_{12}$. Neste trabalho considera-se nula a força de colisão tangencial

\section{VERIFICAÇÃO NUMÉRICA}

Nesta seção o acoplamento DDPMDEM é avaliado numericamente.

O primeiro conjunto de problemas consiste na velocidade terminal de uma partícula. Os parâmetros de cada simulação são aqueles descritos em Mordant e Pinton (2000) e são mostrados na Tabela 2.

Figura 2 - Velocidade terminal para o Caso 1 -

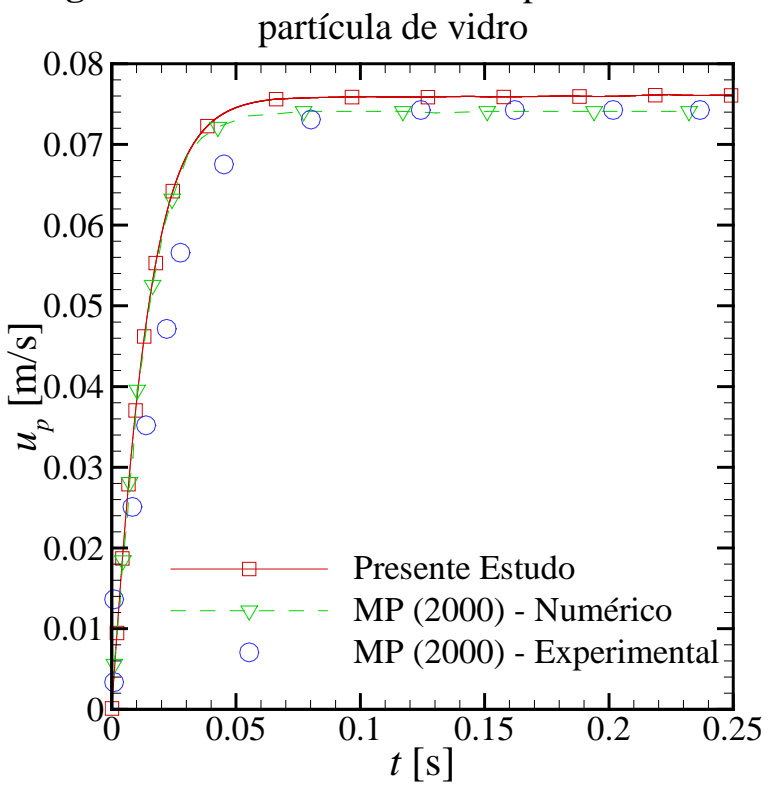


Tabela 2 - Parâmetros empregados para a simulação da velocidade terminal de uma partícula

\begin{tabular}{cccc}
\hline Parâmetro & $\begin{array}{c}\text { Representação } \\
\text { [Unidade] }\end{array}$ & $\begin{array}{c}\text { Caso 1: esfera } \\
\text { de vidro }\end{array}$ & $\begin{array}{c}\text { Caso 2: esfera } \\
\text { de aço }\end{array}$ \\
\hline Massa específica da partícula & $\rho_{p}\left[\mathrm{~kg} / \mathrm{m}^{3}\right]$ & 2560 & 7710 \\
Diâmetro da partícula & $D_{p}[\mathrm{~mm}]$ & 0,5 & 0,8 \\
Velocidade terminal - experimental & $u_{p}[\mathrm{~m} / \mathrm{s}]$ & 0,0741 & 0,315 \\
Massa específica do fluido & $\rho_{\beta}\left[\mathrm{kg} / \mathrm{m}^{3}\right]$ & \multicolumn{3}{c}{998,2} \\
Viscosidade dinâmica & $\mu_{\beta}[\mathrm{Pa} . \mathrm{s}]$ & \multicolumn{2}{c}{$1,003 \cdot 10^{-3}$} \\
\hline
\end{tabular}

Na Figura 2 pode ser observado o resultado para a simulação da velocidade terminal para o Caso 1 e as respectivas comparações com dados numéricos e experimentais de Mordant e Pinton (2000).

É possível notar que existe uma boa concordância entre os resultados numéricos durante toda a região da rampa (onde existe a aceleração da partícula). Após essa região, existe uma pequena diferença entre a velocidade terminal obtida neste trabalho e os dados do modelo numérico e resultados experimentais, devido principalmente pela magnitude do valor da velocidade.

A Figura 3 mostra a comparação dos resultados para a velocidade da partícula ao longo do tempo para o Caso 2, onde considera-se uma partícula de aço.

Figura 3 - Velocidade terminal para o Caso 2 partícula de aço

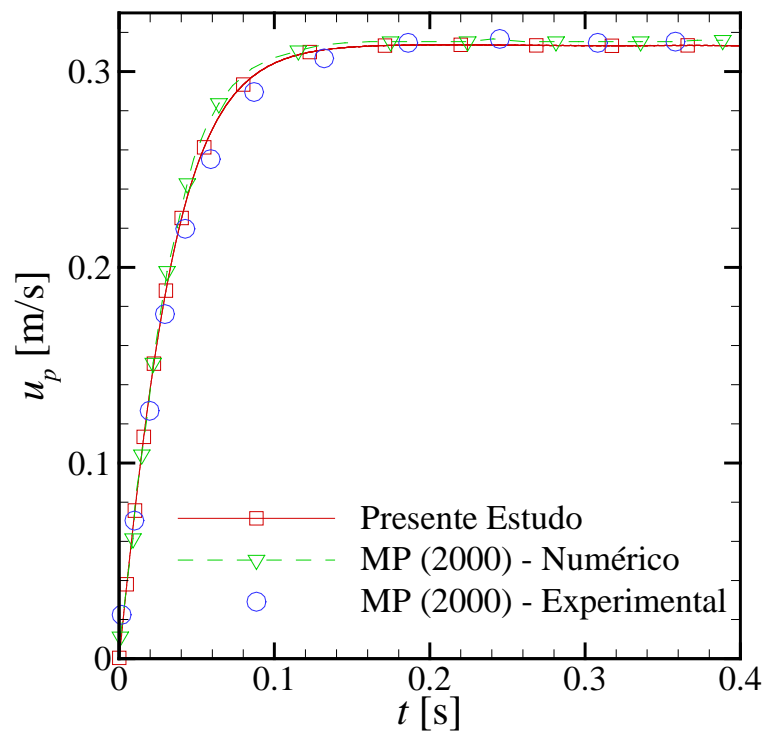

Para avaliar o modelo de colisões adotado, a colisão entre uma partícula e uma parede estática foi analisada segundo os dados experimentais de Gondret et al. (2002).

Os parâmetros empregados na simulação do problema de colisão consistem em $\quad \rho_{p}=7800 \mathrm{~kg} / \mathrm{m}^{3}, \quad D_{p}=3,0 \mathrm{~mm}$, $\rho_{\beta}=1187,6 \mathrm{~kg} / \mathrm{m}^{3}, \quad \mu_{\beta}=0,01$ [Pa.s]. Para caracterizar o momento da colisão, foram empregados quatro coeficientes de restituição $\eta=0,78 ; 0,66 ; 0,45$ e 0,25 .

Na Figura 4 é apresentada a comparação entre o resultado numérico obtido neste trabalho e a literatura disponível.

Figura 4 - Comparação entre os resultados obtidos e o experimento de Gondret et al. (2002)

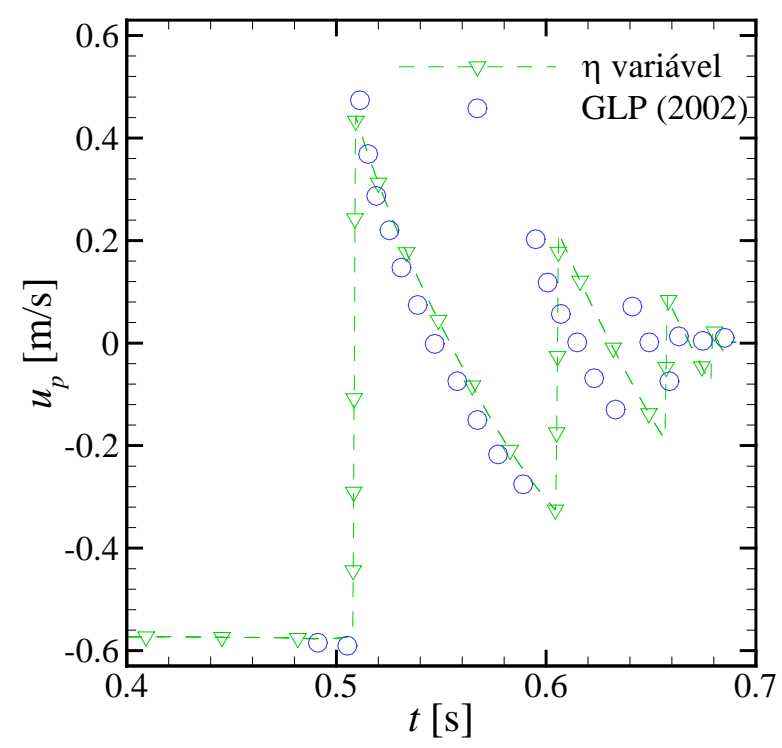

5 RESULTADOS 
Para a análise do processo de vedação considera-se um canal com as seguintes dimensões: $l_{U P}=1,800 \mathrm{~m}, l_{D W}=0,225 \mathrm{~m}$, $h_{C H}=0,045 \mathrm{~m}$. A fratura possui comprimento $h_{F R}=720 \mathrm{~m}$ e espessura $e_{F R}=0,010 \mathrm{~m}$. O fluido utilizado é uma mistura água-glicerina com $\rho_{\beta}=1187,6 \mathrm{~kg} / \mathrm{m}^{3}$ e $\mu_{\beta}=27,973 \cdot 10^{-3}$ Pa.s .

A vazão de fuga $Q_{\text {fuga }}$ é tomada como a relação entre a quantidade de fluido sendo perdido pela fratura ao longo do processo de injeção $q_{\text {fuga }}$ e a vazão de fuga inicial, antes do processo de injeção $q_{\beta, F R, o}$ - Equação (7).

$Q_{\text {fuga }}=\frac{q_{\text {fuga }}}{q_{\beta, F R, o}}$

O monitoramento da pressão na entrada do canal, $P_{\text {ent }}$, é definido como a relação entre pressão durante o processo de injeção de partículas, $p_{m, C H, i}$, e a pressão devido à perda de fluido inicial, $p_{\beta, C H, i}$, assim como mostrado na Equação (8).

$P_{e n t}=\frac{p_{m, C H, i}}{p_{\beta, C H, i}}$

Além da vazão de fuga e da pressão na entrada do canal, as características geométricas do leito de partículas também são monitoradas: o posicionamento $h_{p c t, i}$ e $\mathrm{o}$ comprimento do leito $h_{p c t}$ bem como o preenchimento vertical da fratura $e_{\%, \mathrm{FR}}$, indicados na Figura 5.
Figura 5 - Parâmetros geométricos de monitoramento para o leito de partículas

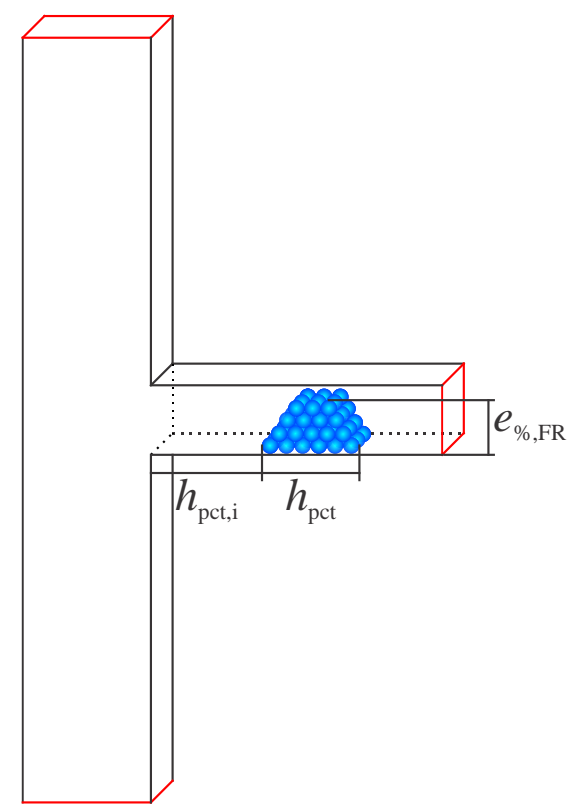

O preenchimento da fratura no canal é analisado através da variação da quantidade de partículas adicionadas ao fluido por segundo $N_{p, i n j}$. Quatro configurações foram avaliadas: $N_{p, i n j}=1000,1500,2000$ e 2500 .

$\mathrm{O}$ resultado do processo de preenchimento da fratura, apresentado na Figura 6, mostra que não existe variação significativa na forma geométrica do leito de partículas. Para evidenciar esse comportamento, a Tabela 3 mostra as características geométricas de cada leito.

Figura 6 - Forma do leito de partículas em relação à quantidade de partículas injetadas por passo de tempo

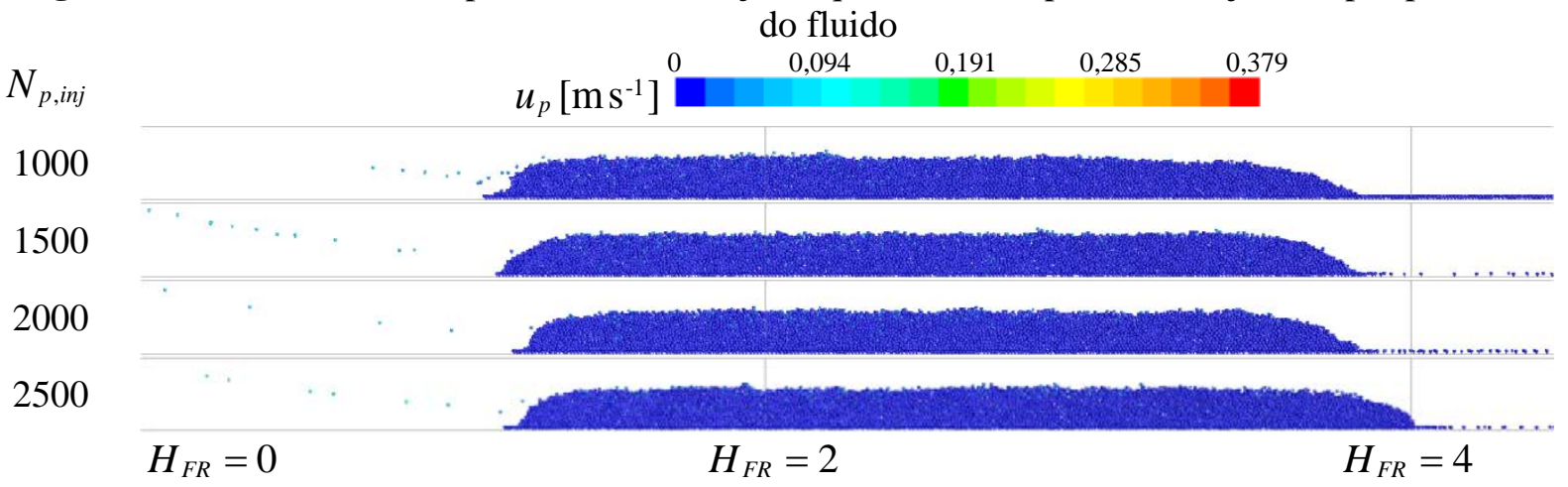


Tabela 3 - Características geométricas referentes

\begin{tabular}{|c|c|c|c|c|}
\hline $\mathrm{N}_{p, i n j}$ & $\begin{array}{l}h_{p c t, i} \\
{[\mathrm{~mm}]}\end{array}$ & $\begin{array}{l}h_{p c t} \\
{[\mathrm{~mm}]}\end{array}$ & $\begin{array}{c}e_{\%, F R} \\
{[\%]}\end{array}$ & $\begin{array}{l}t_{\text {est }} \\
{[\mathrm{s}]}\end{array}$ \\
\hline 1000 & 54 & 117 & 57 & 95 \\
\hline 1500 & 54 & 122 & 57 & 77 \\
\hline 2000 & 57 & 115 & 57 & 62 \\
\hline 2500 & 56 & 124 & 57 & 57 \\
\hline
\end{tabular}

A influência da concentração de partículas é verificada no tempo necessário para promover o preenchimento da fratura, $t_{\text {est }}$ : utilizar uma concentração de partículas elevada colabora com a velocidade de preenchimento da fratura. Para as variações realizadas, observou-se uma melhora de, aproximadamente, $40 \mathrm{~s}$ entre as injeções de 1000 e 2500 partículas por segundo.

A partir do monitoramento da vazão de fuga $Q_{\text {fuga }}$, mostrado na Figura 7, é possível notar que existe um aumento inicial de $Q_{\text {fuga }}$, aqui chamado de overshoot. Esse fenômeno acontece devido à passagem de partículas pela entrada da fratura, mas não colaboram efetivamente com o preenchimento.

Figura 7 - Monitoramento da vazão de fuga ao

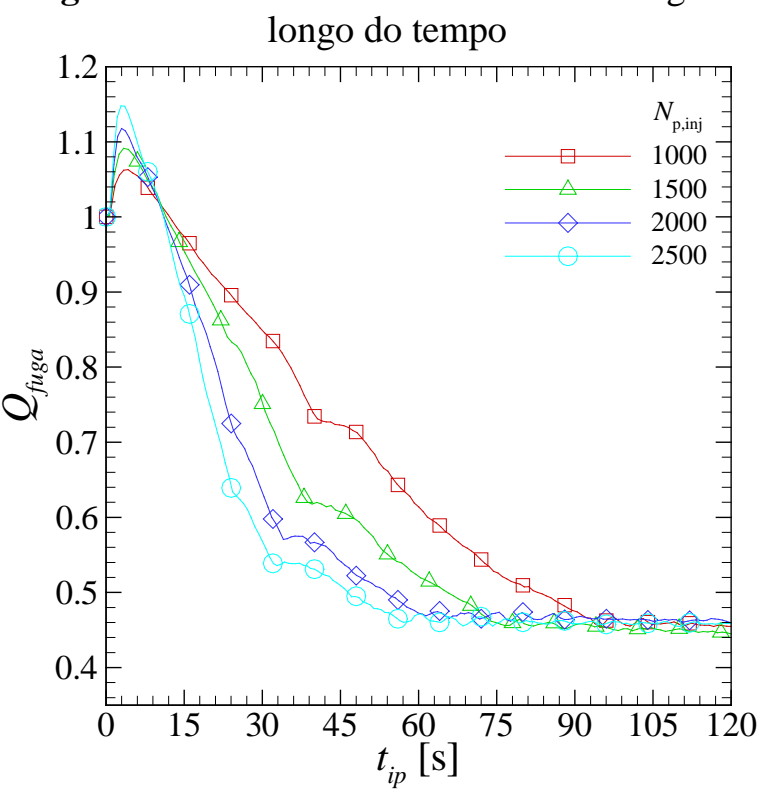

Uma vez que o leito de partículas comece a ser formado no interior da fratura, verifica-se uma subsequente redução da vazão de fuga. O processo continua, até que a vazão se estabilize, indicando o fim do processo de preenchimento.

A injeção de partículas no escoamento causa um aumento associado de pressão na entrada do canal, mostrado na Figura 8.

Figura 8 - Monitoramento da pressão na entrada do canal ao longo do tempo

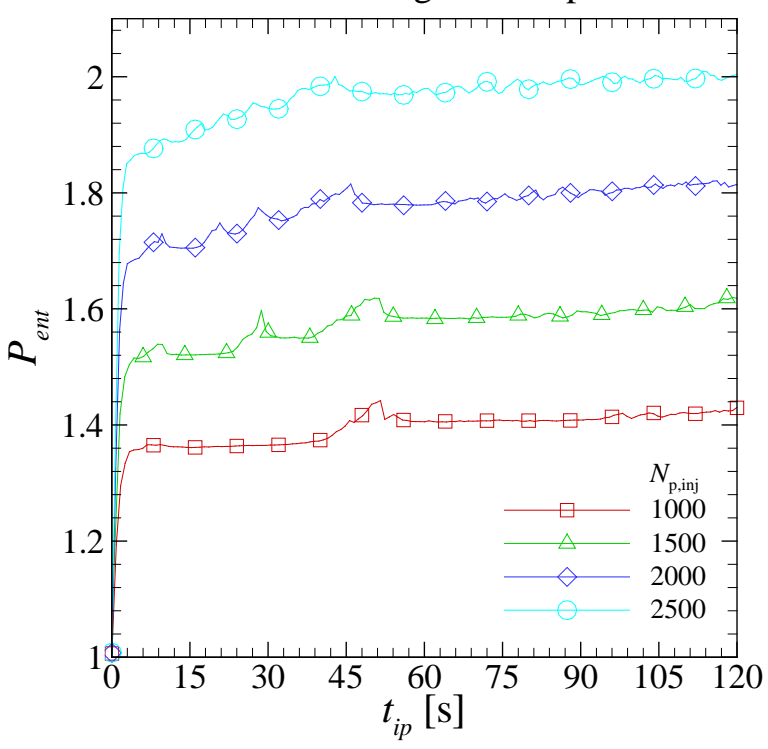

Como esperado, quando a concentração de partículas é maior, o aumento de pressão observado na entrada do canal também é maior, devido à pressão associada à massa de partículas.

Tal aumento de pressão pode ser usado, ainda, para explicar a diferença observada no overshoot da Figura 7: o aumento de pressão causado pela injeção de partículas age no sentido de "expulsar" o fluido pela fratura. Dessa forma, aumentar a concentração de partículas no escoamento implica em aumentar o overshoot observado na Figura 7.

\section{CONSIDERAÇÕES FINAIS}

Neste trabalho a abordagem eulerianalagrangiana para o estudo do escoamento 
particulado aplicado para ao preenchimento de fratura em um canal fraturado foi aplicada.

O preenchimento da fratura foi analisado através da quantidade de partículas sendo injetadas no canal. Foram monitoradas a vazão de fuga devido à presença da fratura, a pressão na entrada do canal fraturado e as características geométricas do leito de partículas.

Verificou-se que a quantidade de partículas adicionadas ao escoamento não influencia nem a vazão de fuga no final do processo de preenchimento nem as características geométricas do leito de partículas formado no interior da fratura.

Por outro lado, aumentar a concentração de partículas no canal acelera o preenchimento da fratura, mas possui a desvantagem de elevar a pressão na entrada do canal.

\section{NOMENCLATURA}

\begin{tabular}{|c|c|}
\hline $\mathbf{N}_{p, i n j}$ & $\begin{array}{l}\text { Quantidade de partículas } \\
\text { por segundo }\end{array}$ \\
\hline$Q_{\text {fuga }}$ & Vazão de fuga \\
\hline$P_{e n t}$ & $\begin{array}{l}\text { Pressão na entrada do } \\
\text { canal }\end{array}$ \\
\hline$h_{p c t, i}$ & $\begin{array}{l}\text { Posição do início do } \\
\text { leito }\end{array}$ \\
\hline$h$ & Comprimento \\
\hline$e_{\%, F R}$ & Preenchimento vertical \\
\hline$e_{F R}$ & Espessura da fratura \\
\hline$l$ & Comprimento \\
\hline$Z_{F R}$ & $\begin{array}{l}\text { Comprimento na direção } \\
\mathrm{z}\end{array}$ \\
\hline$U$ & $\begin{array}{l}\text { Velocidade de entrada } \\
\text { de fluido }\end{array}$ \\
\hline Re & Número de Reynolds \\
\hline$\mu$ & Viscosidade \\
\hline$\rho$ & Massa específica \\
\hline$t$ & Tempo \\
\hline$\varepsilon$ & Fração volumétrica \\
\hline $\boldsymbol{u}$ & Vetor velocidade \\
\hline
\end{tabular}

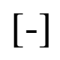

[-]

[m]

[m]

[\%]

[m]

$[m]$

[m]

$\left[m \cdot s^{-1}\right]$

$[-]$

$[\mathrm{Pa} \cdot \mathrm{s}]$

$\left[\mathrm{kg} \cdot \mathrm{m}^{-3}\right]$

[s]

$[-]$

$\left[m \cdot s^{-1}\right]$ $\boldsymbol{p} \quad$ Vetor pressão

$[\mathrm{Pa}]$

g Vetor aceleração da gravidade

F Vetor força

$\mathbf{S}_{D P M}$ Termo fonte

$\boldsymbol{x}$ Vetor posição

$m$ massa

C Coeficiente

$k \quad$ Constante de rigidez

$\delta \quad$ Sobreposição

Coeficiente de

$\gamma$ amortecimento

$\lambda$ Direção normal

$\eta \quad$ Coeficiente de restituição

$q \quad$ Vazão percentual

$p$ Pressão

$\zeta_{12}$ Direção tangencial

$\mu_{a} \quad$ Coeficiente de atrito

$\left[m \cdot s^{-2}\right]$

[N]

$[N]$

[m]

[kg]

[-]

$\left[N \cdot m^{-1}\right]$

[m]

[-]

[-]

[-]

[\%]

[\%]

$[-]$

$[-]$

Subscritos

pct Leito de partículas

$F R \quad$ Fratura

UP Montante

DW Jusante

$\mathrm{CH} \quad$ Canal

$Z_{F R}$

$\beta$

i

Fluido

Superfície de entrada

DPM Acoplamento líquido-sólido

$p \quad$ partícula

d arrasto

$g b \quad$ Gravidade e empuxo

pg Gradiente de pressão

vm Massa virtual

ls Sustentação de Saffman

DEM Colisão

$n$ normal

tangencial

$t$

12

col

Referente à partícula 1 e 2

colisão

fuga Fuga

o Saída 
$\begin{array}{ll}m & \text { mistura } \\ \text { est } & \text { preenchimento }\end{array}$

\section{REFERÊNCIAS}

BARBOSA, M. V. Análise Paramétrica de Escoamento Particulado Aplicado ao Preenchimento de Fraturas. 2015, 136p. Dissertação (Mestrado em Engenharia Mecânica) - Universidade Tecnológica Federal do Paraná, Curitiba, 2015.

CUNDALL, P. A.; STRACK, O. D. L. A discrete numerical model for granular assemblies. Géotechnique, v. 29, n. 1, p.4765, 1979.

DE LAI, F. C. Simulação numérica do escoamento particulado para o preenchimento de canal fraturado. 2013, 201p. Dissertação (Mestrado em Engenharia Mecânica) - Universidade Tecnológica Federal do Paraná, Curitiba, 2013.

GONDRET, P., LANCE, M., PETIT, L.. Bouncing motion of spherical particles in fluids. Physics of Fluids, v. 14, p. 643-652, 2002.

KENDOUSH, A. A., SULAYMON, A. H., MOHAMMEND, S. A. M. Experimental evaluation of the virtual mass of two solid spheres accelerating in fluids. Experimental Thermal and Fluid Science, v. 31, p. 813823, 2007.

LI, A.; AHMADI, G. Dispersion and Deposition of Spherical Particles from Point Sources in a Turbulent Channel Flow. Aerosol Science and Technology, v. 16, p. 209-226, 1992.

LOTH, E. Particles, Drops and Bubbles. Cambridge: Cambridge University Press, 2010.
LUNDING, S. "Collisions \& Contacts between two particles". In: Physics of dry granular media. Dordrecht: Kluwer Academic Publishers, 1998.

MORDANT, N., PINTON, J. F. Velocity measurement of a settling sphere. The European Physical Journal B - Condensed Matter and Complex Systems, v. 18, n.2, p. 343-352, 2000.

MORSI, S.A.; ALEXANDER, A.J. An Investigation of Particle Trajectories in TwoPhase Flow Systems. Journal of Fluid Mechanics, v. 55, n. 2, p. 193-208, 1972.

PATANKAR, N.A.; JOSEPH D.D. Modeling and numerical simulation of particulate flows by the Eulerian-Lagrangian approach. International Journal of Multiphase Flow, v. 27, n. 10, p. 1659-1684, 2001.

POPOFF, B., BRAUN, M. A Lagrangian Approach to Dense Particulate Flows. In: 6th International Conference on Multiphase Flow - ICMF 2007, Leipzig, Germany. Proceedings... Leipzig, Germany, 2007.

YEOH, G. H.; TU, J. Computational Techniques for Multiphase Flows. New York: Butterworth-Heinemann, 2010.

\section{AGRADECIMENTOS}

Os autores agradecem ao apoio do IRF/CENPES/PETROBRAS, ao programa PRH-ANP/MCT (PRH10-UTFPR) e ao Conselho Nacional de Desenvolvimento Científico e Tecnológico (CNPq). 